\title{
Helical vortices generated by flapping wings of bumblebees
}

\author{
Thomas Engels ${ }^{1,4}$, Dmitry Kolomenskiy ${ }^{2}$, Kai Schneider ${ }^{3}$, Marie Farge ${ }^{4}$, \\ Fritz-Olaf Lehmann ${ }^{5}$ and Jörn Sesterhenn ${ }^{1}$ \\ ${ }^{1}$ ISTA, Technische Universität Berlin, Berlin, \\ Müller-Breslau-Strasse 12, 10623 Berlin, Germany \\ ${ }^{2}$ CEIST, Japan Agency for Marine-Earth Science and Technology (JAMSTEC). \\ 3173-25 Showa-machi, Kanazawa-ku, Yokohama Kanagawa 236-0001, Japan. \\ ${ }^{3}$ CNRS, Centrale Marseille, I2M, Aix-Marseille Université, Marseille, \\ 39 rue Joliot-Curie, 13451 Marseille Cedex 20 France \\ ${ }^{4}$ LMD-CNRS, Ecole Normale Supérieure and PSL, Paris, \\ 24 rue Lhomond, 75231 Paris Cedex 05, France \\ ${ }^{5}$ Department of Animal Physiology, Universität Rostock, Rostock, \\ Albert-Einstein-Str. 3, 18059 Rostock, Germany
}

March 21, 2018

\begin{abstract}
High resolution direct numerical simulations of rotating and flapping bumblebee wings are presented and their aerodynamics is studied focusing on the role of leading edge vortices and the associated helicity production. We first study the flow generated by only one rotating bumblebee wing in circular motion with $45^{\circ}$ angle of attack. We then consider a model bumblebee flying in a numerical wind tunnel, which is tethered and has rigid wings flapping with a prescribed generic motion. The inflow condition of the wind varies from laminar to strongly turbulent regimes. Massively parallel simulations show that inflow turbulence does not significantly alter the wings' leading edge vortex (LEV), which enhances lift production. Finally, we focus on studying the helicity of the generated vortices and analyze their contribution at different scales using orthogonal wavelets.
\end{abstract}

\section{Introduction}

Numerical modeling of flapping insect flight receives considerable attention and is motivated by the growing interest in miniaturization of unmanned air vehicles, since flapping wings present a 
bio-inspired alternative to the fixed- and rotary-wings used in human-designed aircraft. The force production in those two kind of fliers relies on entirely different aerodynamic mechanisms. Airplane wings are smooth and use airfoil shapes designed to produce lift from an attached flow which is accelerated on the suction side. Flow separation (stall) limits the range of angle of attack in which these airfoils are useful. By contrast, insect wings feature sharp edges, essentially flat profile and large angles of attack. Under these conditions, flow separation is inevitable and large amounts of vorticity are generated at the leading edge. This vorticity forms a strong vortex which moves with the wing and detaches only at the stroke reversals. It has been suggested that insects can capture it at early times in the following half-stroke to provide an additional benefit [26]. Some insects clap their wings together and the subsequent opening motion creates a fluid jet which also provides additional forces. This mechanism is known as clap-fling-sweep [43] and it has recently been revisited [21]. Dragonflies and some other species can control their four wings independently and have arranged them in a configuration that allows aerodynamic interaction between fore- and hindwing. This interaction depends on the phase difference in their kinematics and can contribute to force production as well [45], [22], [16].

Previous research on the flow generated by flapping wings indicates the important role of the leading edge vortex (LEV) [31, 6]. This vortex has a conical structure due to the three-dimensional motion of the wings. Vorticity is produced at the sharp leading edge, and outwards velocity (from the root to the tip of the wing) develops above the suction surface of the wing, see, e.g., [33, 20, 23]. Such alignment of the vorticity and the velocity has important consequences for the dynamics of the vortex [4. On one hand, the excess vorticity is constantly transported into the wing tip vortex rather than being shed periodically from the leading edge [33]. On the other hand, the swirl angle is large and the vortex can burst [34]. Swirling flows are characterized by strong helicity, which is defined by the scalar product of velocity and vorticity vectors and corresponds to their alignment or anti-alignment. Consideration of the helicity dynamics in flows over flapping or revolving wings can therefore bring important insights into the processes that determine the flow topology.

Helicity has received much attention in the topological fluid dynamics community to measure the linkage and knottedness of vortex lines in the flow. For a review we refer for instance to [37]. In the turbulence community helicity has been used to characterize three-dimensional swirling coherent structures, which correspond to flow regions of maximum helicity [12]. This local alignment or anti-alignement of velocity and vorticity implies that the nonlinear term of the Navier-Stokes equations is depleted and thus the nonlinear energy transfer is slowed down. This energy cascade, also known as Kolmogorov cascade, transfers energy from larger to smaller and smaller scales until it is eventually dissipated. Its inhibition in regions of strong helicity indicates that these structures tend to be more stable and to persist coherently in time [38]. An example for flows with maximum helicity are Beltrami flows, which correspond to eigenfunctions of the curl operator and are hence solutions of the steady Euler equations.

To get insight into the scale distribution of helicity we decompose the velocity and vorticity into 
orthogonal wavelet bases. Wavelets are localized functions in scale and space and allow analyzing flow fields efficiently. Thus the scale-dependent helicity, introduced in [46], can be computed. A review on wavelet based statistical measures for fluid and plasma turbulence can be found in [13].

The aim of this work is to examine the helicity dynamics in flows over model insect wings in connection with the effects that were previously described in terms of the vorticity and the velocity. We propose helicity as a new diagnostics to study the vortices generated by flapping and revolving wings. Although it is often stated that the leading edge vortex is 'helical', its helicity, in the sense of its proper mathematical definition, has received surprisingly little attention. It has only been used to discuss the bursting of the leading edge vortex on a revolving wing, i.e. its transition from a simple to a more complex topology [17]. This transition is reflected as a drop in the volume integral of helicity in the leading edge vortex, but not in the generation of aerodynamic force, as such a burst vortex still induces a locally reduced pressure. We should however stress the difference between vortex bursting, i.e. the change from a simple to a more complex topology, and vortex shedding. In the latter, the leading edge vortex periodically leaves the vicinity of the wing and constitutes a wake, and consequently the aerodynamic forces oscillate, with a significantly reduced mean value [23]. Whether vortex shedding occurs or not depends, amongst others, on the Rossby number and the wing aspect ratio [28, 25]. In a different context, namely the wing/wing interaction of fore- and hindwing in dragonflies, the 'swirl' of the wake has been discussed, [45], but swirl was therein considered as measure for lateral impulse transport rather than the helicity as considered here.

First, we investigate a simplified configuration of an unilaterally rotating bumblebee wing and perform high resolution numerical computations. The flow fields are studied and, in particular, the leading edge vortex is examined. Second, we analyze data of a flapping bumblebee flying in turbulent flow, presented in [9]. We use the orthogonal wavelet decomposition of the flow field to analyze the production of helicity at different scales, which is then quantified by the wavelet spectrum of helicity and its spatial variability.

The manuscript is organized as follows: In section 2 we describe, for reasons of self-consistency, the bumblebee model with rigid wings and the computational set-up. The wing kinematics and parameters can be found in the cited references. The numerical method, which is a Fourier pseudospectral method with volume penalization, is briefly recalled too. The computational results are reproducible as the "FluSI" code is open source [10]. The definition of helicity, together with its spectral decomposition and the scale-dependent helicity using orthogonal wavelets are also given. Computational results for rotating and flapping bumblebee wings are presented and subsequently analyzed in section 3. Conclusions of our findings are drawn in section 4 . 


\section{Bumblebee model, numerical method and helicity}

\section{$2.1 \quad$ Bumblebee model}

In this article, a bumblebee (Bombus terrestris), already used in previous work [9], is chosen among the variety of flying insects as typical representative for medium-size species. Bumblebees are known to be relentless all-weather foragers [44, 41, 5] and thus encounter a wide range of flow conditions from laminar to fully turbulent [9, 5]. The flow they generate while flying remains in a range of Reynolds number which can be computed by direct numerical simulation (DNS) using high-performance computing facilities. The key parameters, which we use in both setups described below, of the model insect are: wing length $R=13.2 \mathrm{~mm}$, wingbeat frequency $f=152 \mathrm{~Hz}$, total mass $m=175 \mathrm{mg}$, forward flight speed $u_{\infty}=2.5 \mathrm{~m} / \mathrm{s}$, Reynolds number $R e=\bar{u}_{\text {tip }} c_{m} / \nu=2060$, where $\bar{u}_{\text {tip }}=8.05 \mathrm{~m} / \mathrm{s}$ is the mean wingtip velocity, $c_{m}=A / R=4.01 \mathrm{~mm}$ the mean chord length, $A=52.96 \mathrm{~mm}^{2}$ the wing surface and $\nu=15 \cdot 10^{-6} \mathrm{~m}^{2} / \mathrm{s}$ the viscosity of air. The planform of the wing is illustrated in Fig. 1 p. The wing is modeled as a rigid, flat surface.

A different definition of the Reynolds number can be based on the mean velocity at the radius

of gyration, $R_{2}=\sqrt{\int_{0}^{R} r^{2} c(r) \mathrm{d} r / A}=7.6032 \mathrm{~mm}$, which yields $R e_{2}=1187$. Using $R_{2}$ has its root in the blade element theory [7], where it appears naturally, and it has been suggested to provide a better value for comparison in the case of revolving wings, as it reflects also the aspect ratio [25]. Note that the velocity at the center of wing area can also be used as reference velocity and it may be advisable for the purpose of comparison between different flappers [32. In this paper, however, we only consider one wing shape. As both Reynolds numbers are common, we will use both definitions.

For the rest of the article we shall only use dimensionless quantities, normalized with a length scale $L=R$, a mass scale $M=\varrho_{f} L^{3}$ (which implies that the dimensionless fluid density is unity) and a time scale $T$, which we choose depending on the setup.

\subsubsection{One revolving wing: the canonical model}

Prior to analyzing the complete insect model, we focus in this part on a commonly used reduced model, which consists of a single, revolving wing. This canonical setup is often used to study the leading edge vortex [23, 15, 14, 17, 25]. We fix the angle of attack to $\alpha=45^{\circ}$ (i.e., the feathering angle, for details see Figure 1 and [10]). The rotation angle varies as

$$
\phi(t)=\dot{\Phi}\left(\tau e^{-t / \tau}+t\right)
$$

which is the same as used in previous work [23]. After a transient time, $\tau=0.4$, the rotation angle grows linearly in time. The wingtip velocity is $u_{\text {tip }}=\dot{\Phi}$ in the steady rotation regime since the wing length is normalized. We choose the time scale such that the wingtip velocity is unity, thus 


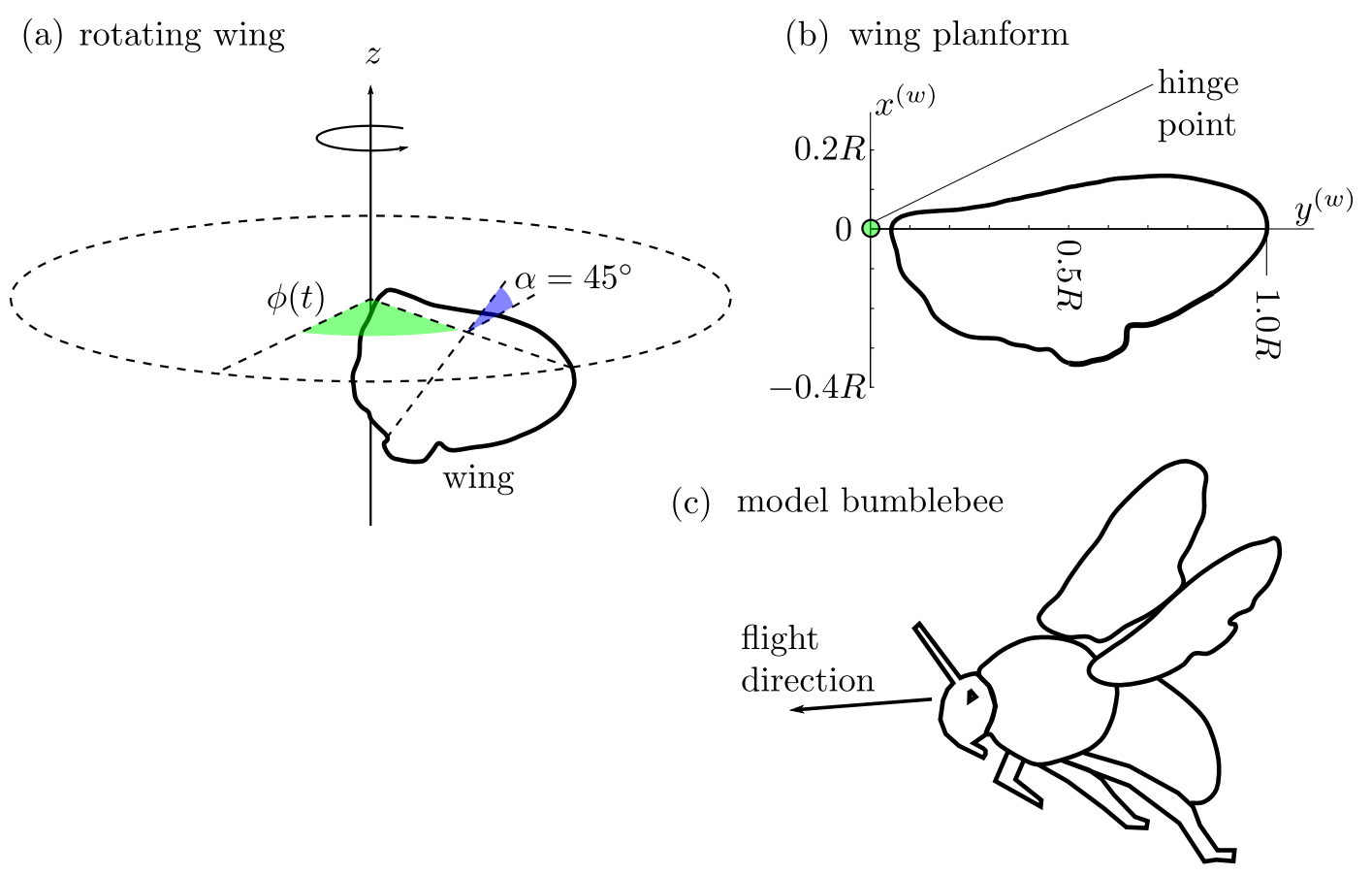

Figure 1: Setup: configuration for the revolving wing (a), wing mask (b) and bumblebee mask (c).

$T=1 / \dot{\Phi}$. The first full rotation would thus be completed at $t=6.68[T]$, but our computations are stopped at $t=6$ to avoid the wing interacting with its own wake. As $t$ and $\phi$ are equivalent, we use $\phi$ as it is more intuitive in this case. The Reynolds numbers based on the terminal velocity of the wing are the same as stated previously for the complete bumblebee, and the Rossby number is defined as $R o=R_{2} / c_{m}$ [28]. In our computations, $R o=1.87$. For comparison, we also perform a viscous simulation in which we multiply the viscosity by ten. Fig. 19 illustrates the setup. The wing revolves around a hinge placed at the center of a domain of size $4 \times 4 \times 2$ wing lengths, which is discretized using $1024 \times 1024 \times 512$ grid points.

\subsubsection{Two flapping wings: the complete model}

The previously described revolving setup is a simplification, and differs in several aspects from an actual bumblebee. As the wing kinematics in flapping flight is a periodic back-and-forth motion, each wingbeat consists of two half strokes, usually termed up- and downstroke. In each half stroke, a new leading edge vortex is created and shed as a vortex puff at the stroke reversal. Describing the precise wingbeat kinematics of insects is beyond the scope of this article, as it depends on species, flight situation and varies between individuals. The wingbeat motion is essentially parallel to the ground in hovering flight, while this stroke plane is more inclined in forward flight. In hovering flight, up- and downstroke are more symmetric than they are in forward flight, which is the case we consider here. The incoming mean flow, which in our simulations accounts for the insects forward flight velocity (Galilean change of reference frame) acts differently on the wing 
during the half-strokes. Nonetheless, in each half stroke, a leading edge vortex is visible, as will be discussed later.

Our simulations take place in a $6 R \times 4 R \times 4 R$ large, numerical wind tunnel, which we resolve with $1152 \times 768 \times 768$ grid points. The insect is tethered - the imposed mean flow accounts for its velocity; its wingbeat kinematics are prescribed. Contrarily to the revolving wing case, we now use the wingbeat duration to normalize time, $T=1 / f$, as this is more natural in the flapping configuration.

To model atmospheric turbulence, we use precomputed homogeneous isotropic turbulence (HIT) as turbulent inflow. The resulting turbulent velocity fluctuations can be added to the laminar inflow in a layer upstream of the insect model. HIT is characterized by the turbulent kinetic energy, the integral length scale and its Reynolds number. We vary the turbulence intensity, $T u=u_{\mathrm{RMS}}^{\prime} / u_{\infty}$ defined as the root mean square of velocity fluctuations normalized to flight velocity, by altering the energy content of the turbulent perturbations superimposed to the mean flow. The entire procedure allows us to study insect flight from laminar to fully-developed turbulent flow regimes. More details on this approach can be found in [9].

\subsection{Numerical method}

Numerical simulations of the flow generated by insects have to face two major challenges. First, as insects fly by flapping their wings, the geometry of the problem is complicated and varies in time, implying that the no-slip boundary condition for the Navier-Stokes equation has to be imposed on a complex fluid-solid interface. Second, many insects can be typically characterized by Reynolds numbers in the intermediate regime [29, 8], i.e., $R e=\mathcal{O}\left(10^{3}\right)$. In this Reynolds number regime, common simplifications, such as the Stokes or inviscid approximations, are essentially nullified, leaving us with the full non-linear unsteady problem. To cope with these challenges, our numerical method combines the volume penalization method [2] with a Fourier pseudospectral discretization [42, 18, for which we developed an open-source computational environment, available on Githubl [10]. The code solves the incompressible, penalized Navier-Stokes equations

$$
\begin{aligned}
\partial_{t} \boldsymbol{u}+\boldsymbol{\omega} \times \boldsymbol{u} & =-\nabla \Pi+\nu \nabla^{2} \boldsymbol{u}-\underbrace{\frac{\chi}{C_{\eta}}\left(\boldsymbol{u}-\boldsymbol{u}_{s}\right)}_{\text {penalization }}-\underbrace{\frac{1}{C_{\mathrm{sp}}} \nabla \times \frac{\left(\chi_{\mathrm{sp}} \boldsymbol{\omega}\right)}{\nabla^{2}}}_{\text {sponge }} \\
\nabla \cdot \boldsymbol{u} & =0 \\
\boldsymbol{u}(\boldsymbol{x}, t=0) & =\boldsymbol{u}_{0}(\boldsymbol{x}) \quad \boldsymbol{x} \in \Omega, t>0,
\end{aligned}
$$

where the mask function $\chi(\boldsymbol{x}, t)$ is unity inside the insect and zero otherwise and $C_{\eta}$ is the penalization constant. The last term is a vorticity damping term used to gradually damp vortices and alleviate the periodicity inherent to the Fourier discretization. The role of this sponge is to relami-

\footnotetext{
${ }^{1}$ https://github.com/pseudospectators/FLUSI
} 
narize the (upwind) flow as does the honeycomb in a windtunnel. Details on how the $\chi$ function and the solid body velocity field $\boldsymbol{u}_{s}$ are constructed can be found in [10], along with a precise description of the insect model and detailed validation tests. We use our code only to compute DNS without additional turbulence modeling, and verify via grid convergence studies that all spatial and temporal scales are resolved. The penalization parameter is set to $C_{\eta}=5.66 \cdot 10^{-4}$, and to determine

$C_{\eta}$ as a function of the other parameters in the simulations, the relation $K_{\eta}=\sqrt{\nu C_{\eta}} / \Delta x=0.074$ is used [10]. This value of $K_{\eta}$ is used in all reported simulations. A sponge layer with a thickness of 32 grid points and a damping constant $C_{\mathrm{sp}}=0.1$ is used to damp the vorticity at the borders of the domain. For comparison, a second simulation is performed increasing the viscosity by a factor of ten, while keeping all other parameters constant. The accompanying paper [10] contains more details on the method, as well as a large variety of validation tests.

\subsection{Helicity, helicity spectra and scale-dependent helicity}

Helicity is a quantity introduced by Betchov in [3], important to study the dynamics of turbulent flows. In [39, 36] it was shown that energy and helicity are two conserved quantities of the incompressible Euler equations. For a comprehensive review on helicity we refer to [37]. Considering the velocity field $\boldsymbol{u}$ and the corresponding vorticity $\boldsymbol{\omega}=\nabla \times \boldsymbol{u}$, the kinetic helicity, $H(\boldsymbol{x})=\boldsymbol{u} \cdot \boldsymbol{\omega}$, can be defined, see, e.g., [39, 36]. The helicity yields a measure of the geometrical statistics of a turbulent flow and allows us to quantify its chirality. It changes sign when applying a mirror symmetry to the reference frame (transforming it from left to right handed). Integrating the helicity over space and dividing it by the volume one obtains the mean helicity $\langle H\rangle=\langle\boldsymbol{u} \cdot \boldsymbol{\omega}\rangle$.

The relative helicity

$$
h(\boldsymbol{x})=\frac{H}{|\boldsymbol{u}||\boldsymbol{\omega}|}
$$

corresponds to the cosine of the angle between the velocity and the vorticity at each spatial position. The range of $h$ thus lies between -1 and +1 , corresponding to anti-alignment and alignment of the velocity and the vorticity vector, respectively.

\section{Energy and helicity balance equation}

Similar to the dissipation of energy (in the absence of forcing), $d_{t}\langle E\rangle=-2 \nu\langle Z\rangle$ where $\langle E\rangle=$ $\left\langle|\boldsymbol{u}|^{2}\right\rangle / 2$ and $\langle Z\rangle=\left\langle|\boldsymbol{\omega}|^{2}\right\rangle / 2$ are respectively the mean energy and enstrophy, mean helicity satisfies a balance equation,

$$
d_{t}\langle H\rangle=-2 \nu\left\langle H_{\omega}\right\rangle
$$

where $\left\langle H_{\omega}\right\rangle=\langle\boldsymbol{\omega} \cdot(\nabla \times \boldsymbol{\omega})\rangle$ is the mean helicity of vorticity (also called superhelicity) assuming absence of helical forcing. In viscous flows, helicity is generated and dissipated, while in the inviscid case $(\nu=0)$ the Euler equations conserve the mean kinetic helicity. Contrary to energy neither 
helicity of velocity nor helicity of vorticity are positive definite quantities. The point-wise helicity $H(\boldsymbol{x}, t)$ of velocity satisfies the equation [24],

$$
\partial_{t} H+\boldsymbol{u} \cdot \nabla H=-\nabla \cdot(\boldsymbol{\omega} p)+\frac{1}{2} \nabla \cdot\left(\boldsymbol{\omega}|\boldsymbol{u}|^{2}\right)+\nu\left(\nabla^{2} H-2(\nabla \boldsymbol{u} \nabla \boldsymbol{\omega})\right)
$$

This shows that for the helicity dynamics both the nonlinear and the viscous terms locally play a role, either in enhancing or diminishing the helicity.

\section{Energy and helicity spectrum}

Computing the Fourier transform of the velocity and the vorticity, denoted by $\widehat{\cdot}$, the isotropic energy and helicity spectra can be defined,

$$
E(k)=\frac{1}{2} \sum_{k=|\boldsymbol{k}|}|\widehat{\boldsymbol{u}}(\boldsymbol{k})|^{2} \quad, \quad H(k)=\sum_{k=|\boldsymbol{k}|} \widehat{\boldsymbol{u}}(\boldsymbol{k}) \cdot \widehat{\boldsymbol{\omega}}(-\boldsymbol{k}) .
$$

Note that $H(k)$ is also real valued, but a signed quantity, and by construction we have $\sum_{k \geq 0} E(k)=$ $E$ and $\sum_{k \geq 0} H(k)=\langle H\rangle$ which justifies that $E(k)$ and $H(k)$ are called the spectral density of energy and helicity, respectively. Applying the Cauchy-Schwarz inequality, it follows that $|H(k)| \leq$ $2 k E(k)$, which motivates the introduction of the relative helicity spectrum $|H(k)| /(2 k E(k)) \leq 1$. In [24] it has been shown to fall off linearly in wave-number for large $k$, restoring thus the mirror symmetry of the flow at small scales in the case of isotropic turbulence.

\section{Scale-dependent energy and helicity}

The vorticity and velocity field can be decomposed into an orthogonal wavelet series, i.e. for the velocity we have

$$
\boldsymbol{u}=\sum_{\mu} \sum_{i} \sum_{j} \widetilde{\boldsymbol{u}}_{\mu, i, j} \psi_{\mu, \boldsymbol{i}, j}(\boldsymbol{x})
$$

where $j$ is the scale index, $\mu$ the direction index and $i$ is the position vector. The coefficients $\widetilde{\boldsymbol{u}}_{\mu, \boldsymbol{i}, j}=\langle\boldsymbol{u}, \psi\rangle$ are then called the wavelet transform of $\boldsymbol{u}$, where $\psi$ is the wavelet. Orthogonal wavelets typically do not posses a closed-from expression, but they are rather defined in terms of quadrature-mirror filters. The contributions at scale $j$ can be obtained (for details see, e.g., [13]) by summing over all scales and directions for a given scale $j$ :

$$
\boldsymbol{u}_{j}(\boldsymbol{x})=\sum_{i} \sum_{\mu} \widetilde{\boldsymbol{u}}_{\mu, i, j} \psi_{\mu, i, j}(\boldsymbol{x})
$$

which corresponds essentially to bandpass filtering since all other scales are set to zero. For the vorticity the above decomposition can be applied analogously. The scale-dependent energy can thus be defined as

$$
E_{j}(\boldsymbol{x})=\frac{1}{2} \boldsymbol{u}_{j}(\boldsymbol{x}) \cdot \boldsymbol{u}_{j}(\boldsymbol{x})
$$


and integrating over $\boldsymbol{x}$ yields the mean energy $\left\langle E_{j}\right\rangle$ at scale $2^{-j}$, which is called energy scalogram. Summing $\left\langle E_{j}\right\rangle$ over scale we obtain the total energy $E=\sum_{j}\left\langle E_{j}\right\rangle$.

Analoguously the scale-dependent helicity can be defined as

$$
H_{j}(\boldsymbol{x})=\boldsymbol{u}_{j}(\boldsymbol{x}) \cdot \boldsymbol{\omega}_{j}(\boldsymbol{x})
$$

which was introduced in [46] in the context of isotropic turbulence. The scale-dependent helicity preserves Galilean invariance, though the kinetic helicity itself does not. Integrating $H_{j}$ over $\boldsymbol{x}$ yields the mean helicity $\left\langle H_{j}\right\rangle$ at scale $2^{-j}$, which we call helicity scalogram. The corresponding mean helicity is obtained by summing $\left\langle H_{j}\right\rangle$ over scale, $\bar{H}=\sum_{j}\left\langle H_{j}\right\rangle$, due to the orthogonality of the wavelet decomposition.

The scale-dependent relative helicity can be defined correspondingly as

$$
h_{j}(\boldsymbol{x})=\frac{H_{j}}{\left|\boldsymbol{u}_{j}\right|\left|\boldsymbol{\omega}_{j}\right|}
$$

and can be used to analyze the probability distribution of the cosine of the alignment angle [46].

The scale $2^{-j}$ can be related to the wavenumber $k_{j}$ as

$$
k_{j}=k_{\psi} 2^{j}
$$

where $k_{\psi}=\int_{0}^{\infty} k|\hat{\psi}(k)| d k / \int_{0}^{\infty}|\widehat{\psi}(k)| d k$ is the centroid wavenumber of the chosen wavelet $\left(k_{\psi}=\right.$ 0.77 for the Coiflet 12 used here). Thus the scale-dependent energy and helicity can be directly related to their corresponding Fourier spectra.

The wavelet energy spectrum can be obtained using the scalogram and eq. (11),

$$
\widetilde{E}\left(k_{j}\right)=\frac{1}{2 \Delta k_{j}}\left\langle E_{j}\right\rangle,
$$

where $\Delta k_{j}=\left(k_{j+1}-k_{j}\right) \ln 2$ [35, 1]. It is thus directly related to the Fourier energy spectrum and yields a smoothed version [11, 35]. The orthogonality of the wavelets with respect to scale and direction guarantees that the total energy is obtained by direct summation, $E=\sum_{j} \widetilde{E}\left(k_{j}\right)$.

The wavelet helicity spectrum can then be obtained likewise

$$
\widetilde{H}\left(k_{j}\right)=\frac{1}{2 \Delta k_{j}}\left\langle H_{j}\right\rangle
$$

and again summation over $j$ yields the total mean helicity. We anticipate that the wavelet helicity spectrum is a smoothed version of the Fourier helicity spectrum.

The spatial variability of the wavelet energy and helicity spectra at a given wavenumber $k_{j}$ can 
be quantified by the standard deviation, defined as

$$
\sigma\left[E_{j}\right]=\frac{1}{2 \Delta k_{j}} \sqrt{\left\langle\left(\boldsymbol{u}_{j} \cdot \boldsymbol{u}_{j}\right)^{2}\right\rangle-\left(E_{j}\right)^{2}} \quad, \quad \sigma\left[H_{j}\right]=\frac{1}{2 \Delta k_{j}} \sqrt{\left\langle\left(\boldsymbol{u}_{j} \cdot \boldsymbol{\omega}_{j}\right)^{2}\right\rangle-\left(H_{j}\right)^{2}} .
$$

Thus the flow intermittency can be quantified. This is not possible using Fourier spectra as all spatial information is lost. The spatial variability of the energy spectrum can be related to the scale-dependent flatness, defined as the ratio of the fourth- to the second-order moment of the scale dependent velocity, as discussed, e.g., in [13]. Increasing flatness values for decreasing scale, i.e., values larger than three which are obtained for a Gaussian distribution, are attributed to the flow intermittency.

\section{$3 \quad$ Numerical results}

The results in this section are all presented in dimensionless form, using the winglength $R$, the mass scale $\varrho_{f} R^{3}$ (therefore the dimensionless fluid density is unity) and a time scale $T$. The latter depends on the context: in the revolving wing, we set $u_{\text {tip }}$ to 1 and thus $T=R / u_{t i p}$, while in the bumblebee setup the cycle duration is a more convenient parameter for normalization.

\subsection{Flow generated by a revolving bumblebee wing}

This section deals with a flow generated by a bumblebee wing which steadily revolves around a hinge point with a constant angle of attack, see Fig. 1 (a). The setup is inspired by experimental contributions considering revolving wings of either rectangular [17] or insect-inspired shape [40]. In particular, [17] focuses on the bursting of the leading edge vortex, i.e. its transition from simple to complex topology. The authors find that the bursting does not have an impact on the aerodynamic

force. The vortex can burst but still remains attached to the wing, i.e., there is no LEV separation, and helicity can be used to characterize this bursting.

We first visualize, in Fig 2, the helicity $H(\boldsymbol{x})$ and vorticity magnitude $|\boldsymbol{\omega}(\boldsymbol{x})|$, at three different instants, for two different flows, corresponding to $R e=206$ and 2060, respectively. For both flows the wing motion starts from rest in a quiescent fluid and a vortex is formed.

The first time instant, $\phi=36^{\circ}$ (top row), corresponds to the early phase of steady rotation. In the left part of Fig. 2, the simulation with $R e=206$ is visualized and the right part corresponds to the $R e=2060$ case, which is the Reynolds number of the bumblebee. In both simulations, large amounts of vorticity are created at the leading edge, where the flow separates due to the elevated angle of attack. Thus, in both cases, an LEV is formed, but the quantitative scale for vorticity is reduced in the viscous case. A tip vortex forms as well in both cases.

At the same time, the visualization of kinetic helicity show that virtually no helicity is generated at the leading edge, even though large amounts of vorticity are available. This indicates the lack of 


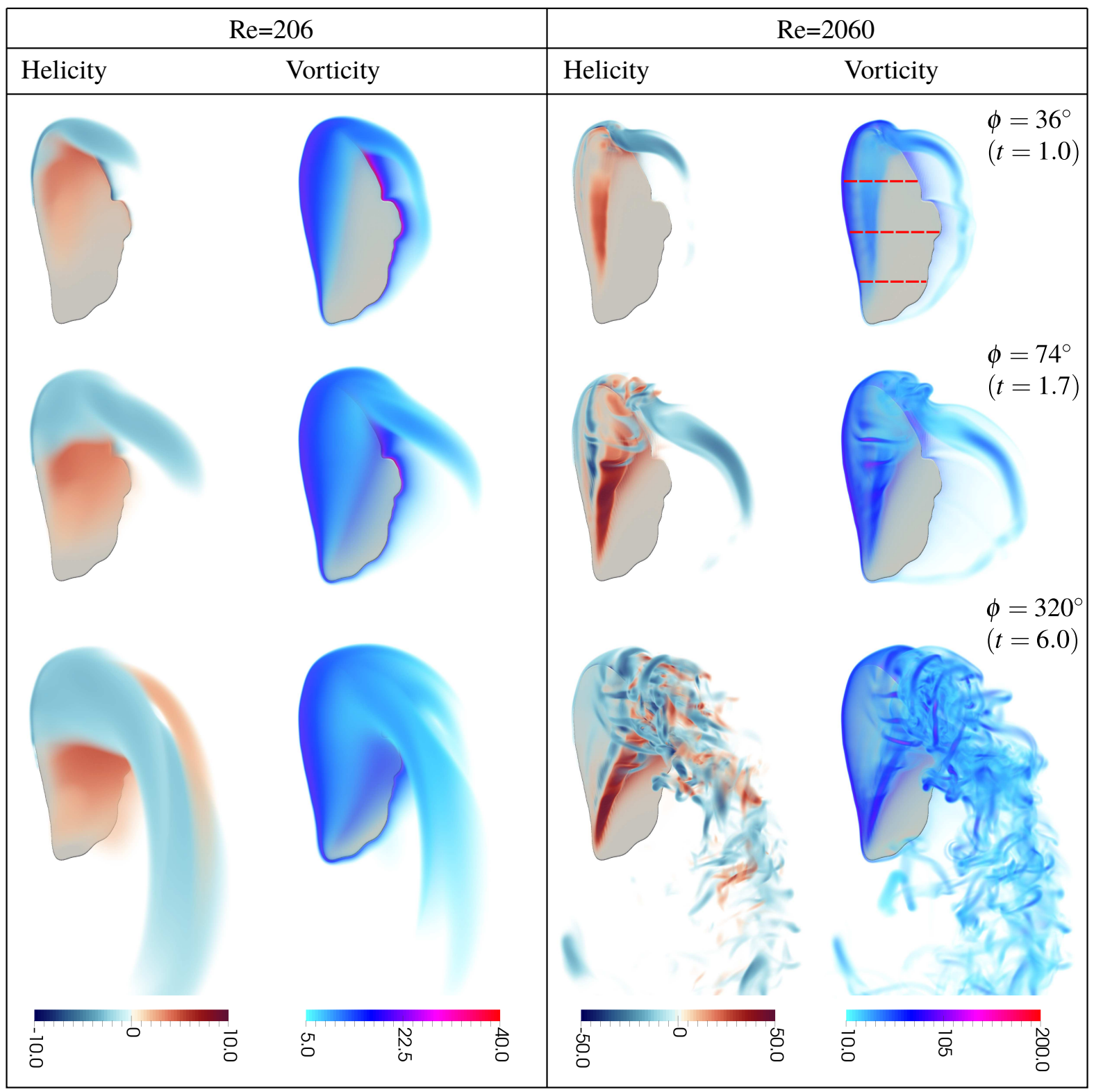

Figure 2: Flows generated by a rotating wing at two different Reynolds numbers, $R e=206$ (left) and 2060 (right), visualized by their helicity $H(\boldsymbol{x})$ and vorticity magnitude $|\boldsymbol{\omega}(\boldsymbol{x})|$ at three rotation angles $\phi$ (rows). The view is rotated such that the observer looks in the direction of the wing normal. The flow topology becomes more complex when the Reynolds number increases. Essential features, such as leading edge and tip vortices, are observed in both cases, but remain stable at $R e=206$ and develop strong instabilities at $R e=2060$. All quantities are dimensionless, i.e. helicity is given in $\left[L / T^{2}\right]$ and vorticity magnitude in $[1 / T]$. Red dashed lines correspond to positions of 2D slices shown in Fig. 4. 
spanwise flow at the leading edge. The axial flow seems to develop further away form the leading edge, which is also where Fig. 2 shows positive helicity, again at different magnitudes for the two Reynolds numbers. In the higher Reynolds number case, the region of positive helicity is more strongly confined and marks a distinct vortex core. In both cases, the wing tip vortex features negative helicity. The topological reconnection of the LEV and the tip vortex contains a curious transition from the positive helicity in the LEV core to the overall negative helicity in the wing tip vortex.

The outwards axial flow in the LEV is driven by the centrifugal force and the axial pressure gradient produced the conical shape of the vortex. The axial flow in the wing tip vortex is created by entrainment of the fluid behind the moving wing. Consequently, the helicity changes sign near the wing tip.

Later on, at $\phi=320^{\circ}$ (bottom row), the differences in the vorticity fields of the two cases become quite remarkable, as the higher Reynolds number case develops much finer flow features near the wing tip, which are inhibited by the viscosity in the other case. It is also noted that a coherent leading edge vortex is visually less easily defined in the low viscosity case. The visualization of kinetic helicity $H(\boldsymbol{x})=\boldsymbol{u} \cdot \boldsymbol{\omega}$ in Fig. 2 looks qualitatively similar to the vorticity magnitude regarding the appearance of fine structures. The tip vortex is helical with a negative value of $H$, while the region near the root until midspan features positive values of $H$. In the high Reynolds case, a strongly helical leading edge vortex is visible at $\phi=75^{\circ}$, which becomes incoherent towards the tip. At $\phi=320^{\circ}$, more than half of the wing features an incoherent, burst leading edge vortex. We note at either Reynolds number that no vortex shedding occurs, meaning that the leading edge vortex remains attached to the wing.

This LEV bursting becomes more clearly visible when integrating the helicity density over a control volume above the suction side of the wing, where the leading edge vortex is found. This value is shown in Fig. 3 (top). From vanishing helicity due to the quiescent initial condition, the integral value $H_{\mathrm{top}}=\iiint_{\Omega_{\mathrm{top}}} H(\boldsymbol{x}) \mathrm{d} V$ follows a qualitatively different evolution for the two Reynolds numbers considered. In the viscous case, $H_{\text {top }}$ is negative throughout the simulation and builds up until around $\phi=180^{\circ}$, remaining constant around -0.08 afterwards. By contrast, the high Reynolds number flow first builds up positive $H$ until a maximum is reached at $\phi=81^{\circ}$, then rapidly drops to a constant, negative value very close to the viscous case. The breakdown of positive helicity is a consequence of vortex bursting.

As emphasized in [17, the consequences for the force production are marginal. Fig. 3 (bottom) shows the lift and drag component of the aerodynamic force, which is computed as $\boldsymbol{F}=$ $\int \chi\left(\boldsymbol{u}-\boldsymbol{u}_{s}\right) / C_{\eta} \mathrm{d} V$ [2, 10]. Their evolution with the rotation angle is qualitatively similar, and an almost steady force is produced after $\phi=90^{\circ}$, with only small fluctuations in the higher Reynolds number case. The qualitatively very different behavior in $H_{\text {top }}$ is thus not reflected in the force production. Interestingly, though we varied the Reynolds number by a factor of ten, the lift force changes only by $16 \%$, but the viscous case produces quantitatively more drag than lift, 

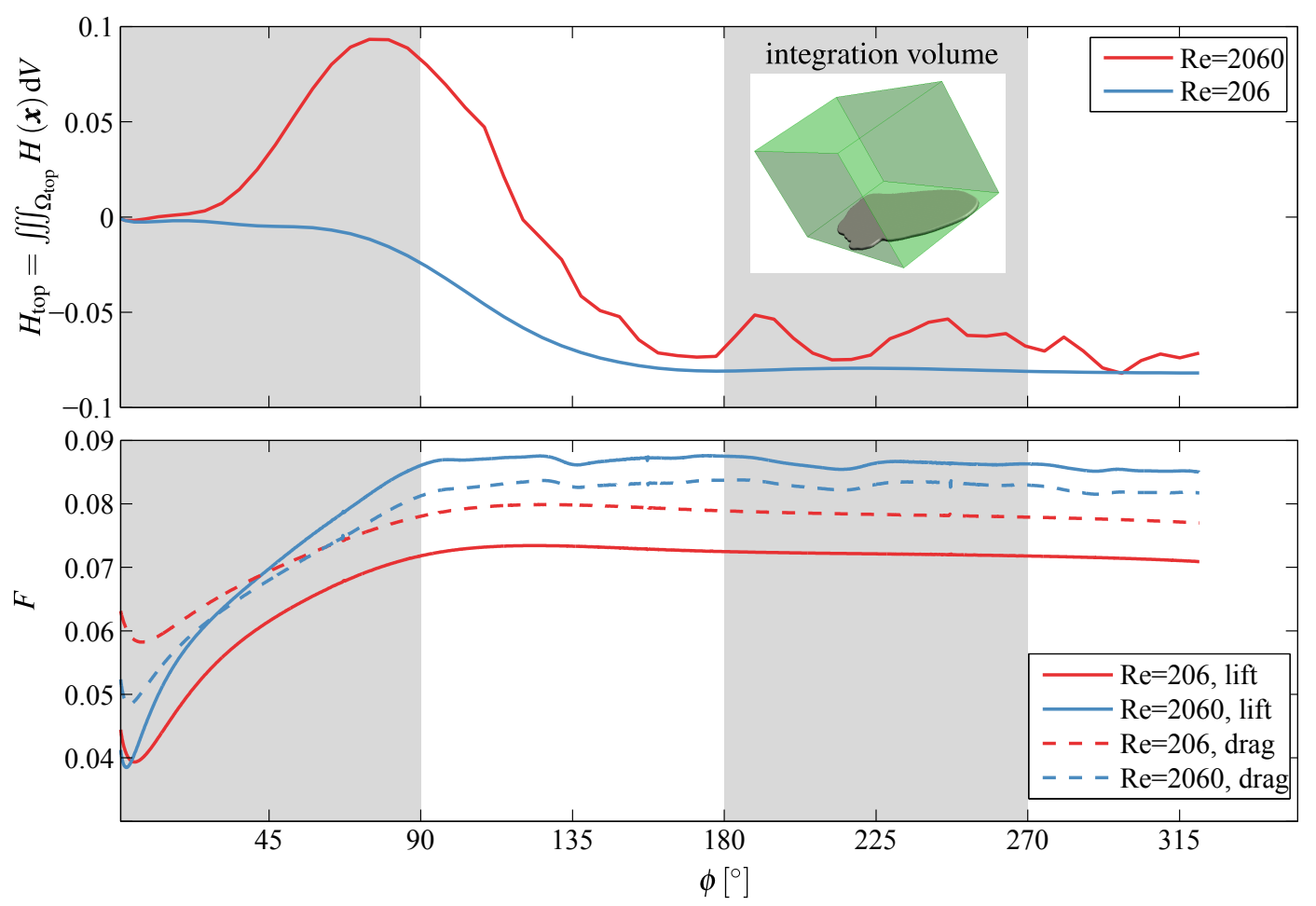

Figure 3: Evolution of helicity, lift and drag as a function of the rotation angle $\phi$ for the revolving wing at $R e=206$ and 2060. Top part shows integral helicity $H_{\text {top }}=\iiint_{\Omega_{t o p}} H(\boldsymbol{x}) \mathrm{d} V$, where $\Omega_{\mathrm{top}}=[-0.35,+0.15] \times[0,1] \times[0,1]$ is a cubic control volume on the top surface (suction side) of the wing, as shown in the inset. Bottom part shows the aerodynamic force, split into lift (in the $z$-direction of the laboratory system) and drag (the magnitude of the $x$-and $y$ component). All quantities are dimensionless, i.e. $F$ is given in $\left[M L / T^{2}\right]$ and $H_{\text {top }}$ in $\left[L^{4} / T^{2}\right]$.

which is the opposite of the higher Reynolds number case.

Figure 4 displays two-dimensional sectional plots of kinetic helicity, axial flow and axial vorticity at three time rotation angles for three different spanwise positions. At $\phi=36^{\circ}$, we observe the formation of a conical LEV core above the suction side of the wing at all three spanwise locations. Large positive spanwise vorticity in the core is collocated with large outwards spanwise velocity, yielding large positive helicity density. In the LEV feeding sheet, however, the helicity is already changing sign from positive over the proximal part to negative over the distal part of the wing. This may be an early sign of the developing breakdown instability. At $\phi=74^{\circ}$, the proximal part of the wing still supports a compact conical core. However, the LEV core bursts over the distal part. This is seen by thickening of the core and and emergence of smaller secondary structures that wrap around the primary core. The helicity is still positive, but not as large as before the burst.

At the final rotation angle, $\phi=320^{\circ}$, the LEV is in its statistical equilibrium state. It begins as a laminar conical vortex from the root of the wing and bursts at around $2 / 3$ of the wing length, forming a series of $3 \mathrm{~d}$ strongly helical trailing vortices (ribs) which are perpendicular to the LEV.

Note that our results are essentially not frame dependent, because the vorticity associated with 


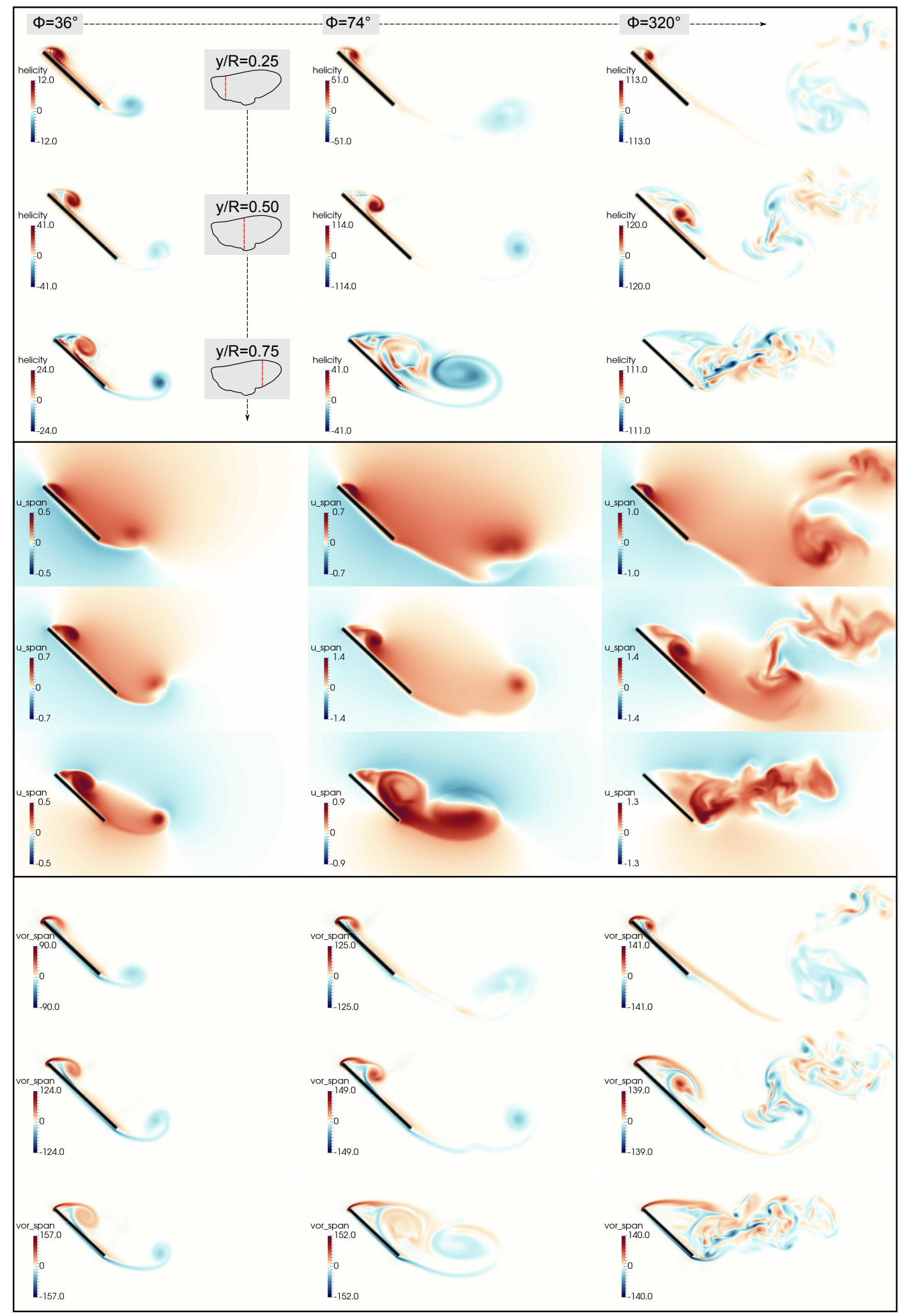

Figure 4: Flow around a rotating wing, $R e=2060$. Figure shows two-dimensional slices of helicity (top box), spanwise velocity (middle box) and spanwise vorticity (bottom box). Slices are at three different spanwise positions, $y / R=0.25,0.50,0.75$ (rows, visualized by insets in top part) and rotation angles $\phi=36^{\circ}, 74^{\circ}, 320^{\circ}$ (columns). All quantities are dimensionless, i.e. helicity is given in $\left[L / T^{2}\right]$, velocity in $[L / T]$ and vorticity in $[1 / T]$. 

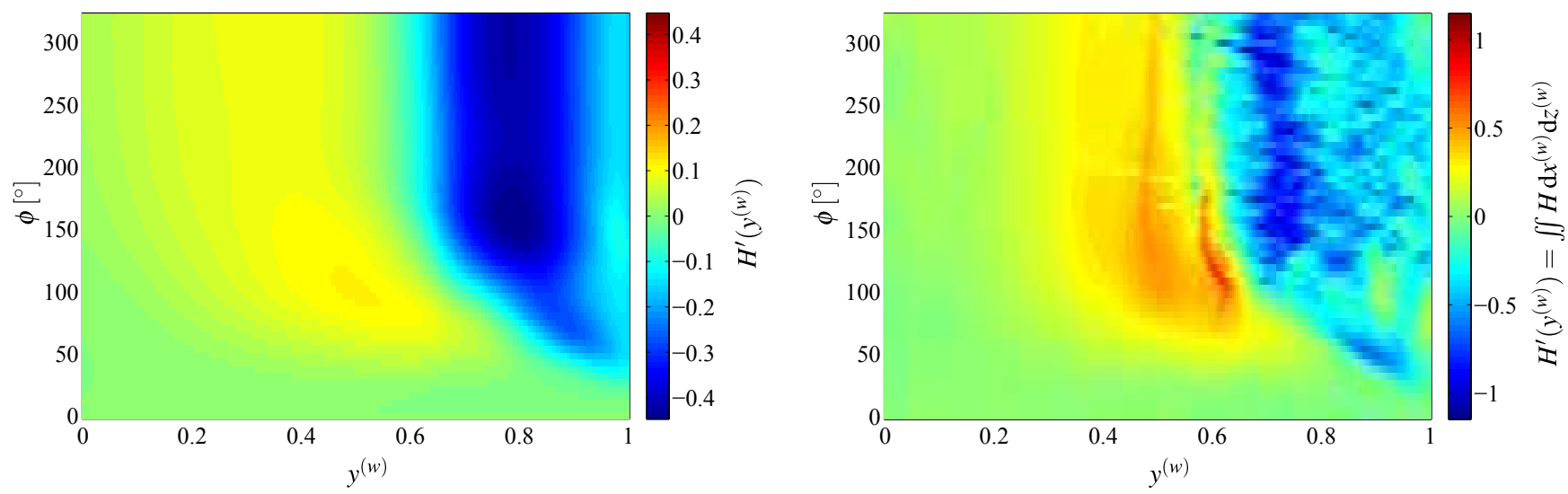

Figure 5: Helicity $H^{\prime}=\iint_{\Omega} H \mathrm{~d} x^{(w)} \mathrm{d} z^{(w)}$, which is the integral of helicity $H(\boldsymbol{x})$ in slices normal to the wing in chordwise direction $\left(y^{(w)}=\right.$ const, see Fig. 1 $\mathrm{b}$ for axis definition), as a function of the distance to wing root $y^{(w)}$ and rotation angle $\phi$. The integration domain is $\Omega=[-0.35,0.15] \times[0,1]$. All quantities are dimensionless, i.e. $y^{(w)}$ is given in $[L]$.

changing between the laboratory reference frame and a moving reference frame of the wing is of order 1 , but the vorticity in the vortices is of order 100, i.e., two orders of magnitude larger.

Garmann and Visbal [14] point out the co-existence of the burst instability of the LEV core and the Kelvin-Helmholtz instability in the feeding LEV sheet. While the LEV burst is obvious in our numerical simulations, the Kelvin-Helmholtz instability is not apparent, possibly because the shear layer transition point is too far from the rotation axis at $R e=2060$. The two instabilities may have different scaling with the Reynolds number, and this question needs further investigation.

Figure 5 illustrates the evolution of relative helicity as a function of spanwise position for $R e=206$ (left) and $R e=2060$ (right). The horizontal axis in each of the panels corresponds to the spanwise position $y^{(w)}$ (see Fig. 1b for the axis definition), and the vertical axis corresponds to the rotation angle $\phi$. Thus, the color of a selected row of pixels on the diagram shows how the helicity density varies along the wing at a given $\phi$. A column of pixels, by contrast, shows how the helicity density at a given $y^{(w)}$ varies in time. We first discuss the low Re case. At startup, $\phi<25^{\circ}$, the helicity density is negligibly small, which means that, even though some strong vorticity may be produced at the sharp edges, no significant axial flow has developed in the vortex cores. After $\phi=25^{\circ}$, the positive helicity builds up in the LEV, and negative helicity builds up in the wing tip vortex. The wing tip vortex expands as time progresses, until saturation after $\phi=150^{\circ}$.

At the larger Re, the diagram is similar to the extent that helicity is positive in the LEV, negative in the wing tip vortex, and the two regions develop in time until saturation at about the same time $\phi=150^{\circ}$ and the same radial position $y^{(w)}=0.55$. However, the magnitude of helicity is about 3 times as large compared to the low-Re case. This is probably related to the enhanced axial flow in the high-Re LEV, and overall larger vorticity production in that case.

The wavelet energy spectra (Fig. 6, left) in log-log representation and helicity spectra in lin-log 

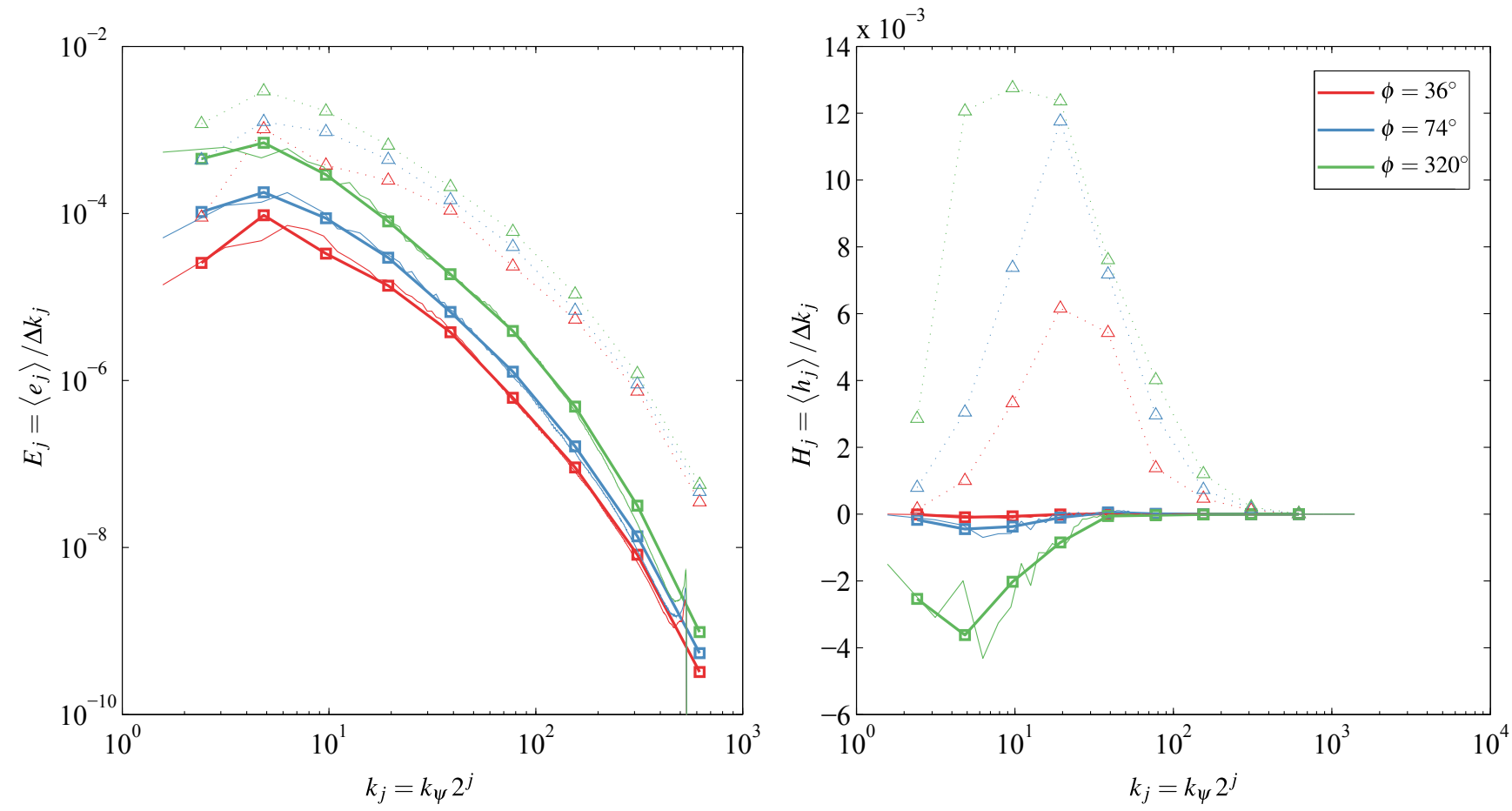

Figure 6: Scalograms of energy and helicity in the rotating wing at $R e=2060$ : Wavelet energy (left) and helicity (right) spectra (thick, continuous lines) together with their corresponding standard deviation (dashed lines) at three different rotation angles, $\phi=36^{\circ}, 74^{\circ}$ and $320^{\circ}$, computed using orthogonal Coiflet 12 wavelets. For comparison, the Fourier spectra are also shown (thin, continuous lines).

representation (Fig. 6, right) show the scale distribution of energy and helicity, respectively. They yield similar information as the Fourier spectra, however the wavelet spectra are less influenced by the mask function $\chi(\boldsymbol{x}, t)$, in particular at small scales, used in the computations to impose the no-slip boundary conditions. We observe that both energy and helicity values grow in time and that the maximum magnitude is at the same wavenumber, $k=5$, where also a peak in the kinetic energy is observed. The corresponding standard deviations (dashed lines) illustrate the spatial fluctuations of energy and helicity. We find that small energy and helicity values at large $k$ exhibit nevertheless large fluctuations, which is a signature of the flow intermittency.

Visualizations of the scale-wise helicity together with the energy are presented in Figure 7 at $t=6$. The (positively) helical leading edge vortex is well visible at scales $2^{-5}$ and $2^{-6}$, while the tip vortex, visible at larger scales, is predominantly negative. However, positively helical structures are also present in the tip vortex at all scales. Also, a negatively helical secondary LEV core is visible, adjacent to the primary positive LEV at scales $2^{-6}$ and $2^{-7}$. The secondary core is rotating in opposite direction of the primary core, see, e.g., [14]. Fine scaled energy contributions are located near the wing, while the far field features energy at relatively larger scales. This is not surprising, since vortical structures at smaller scales decay faster because of viscous dissipation. 


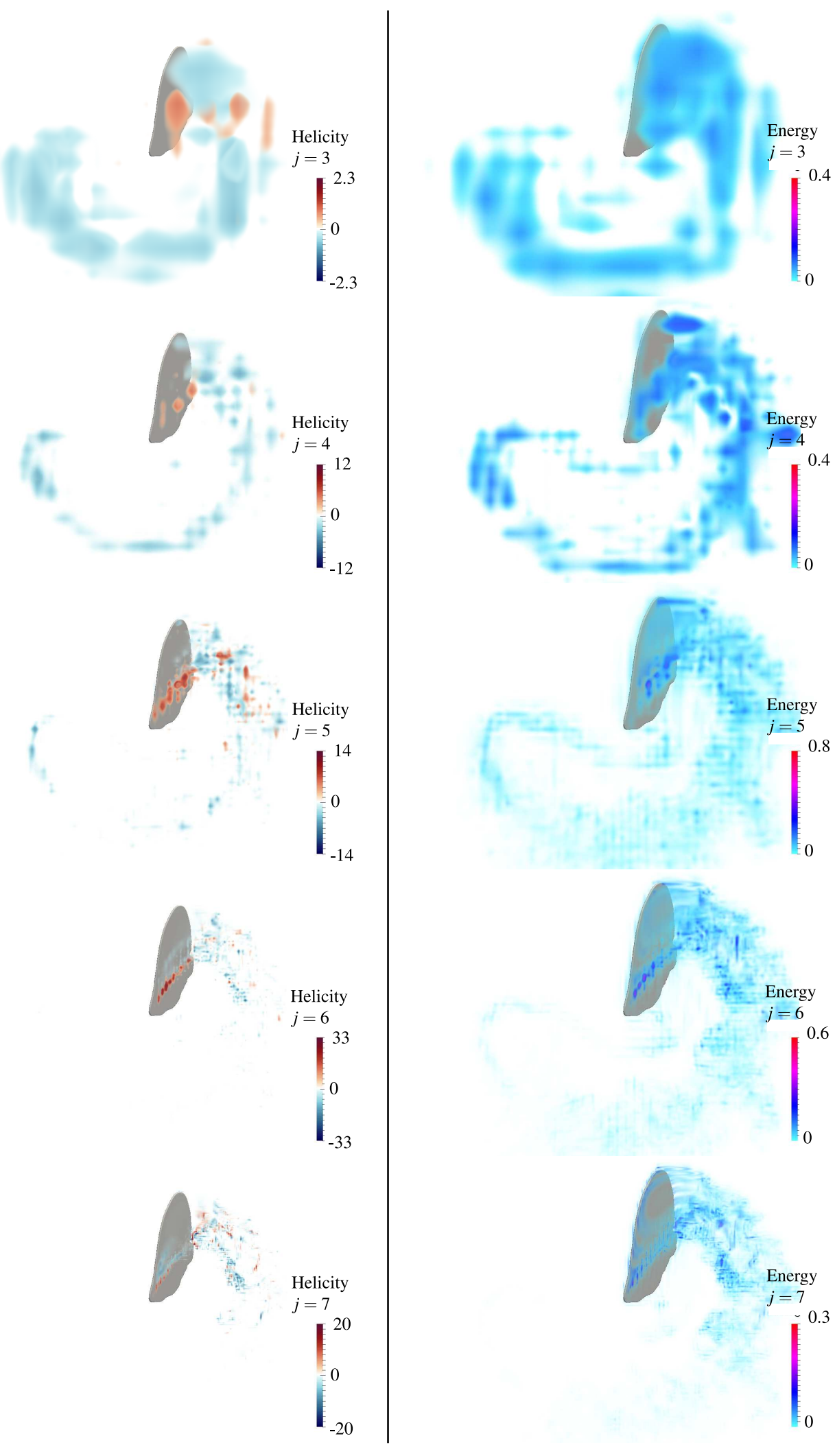

Figure 7: Rotating wing at $R e=2060$ and the terminal rotation angle $\phi=320^{\circ}$. Helicity (left) and energy (right) are visualized at four different scales from $j=3$ (large scale) to $j=7$ (small scale) (from top to bottom). Coiflet 12 orthogonal wavelets were used for scale extraction. 


\subsection{Flow generated by flapping wings of a tethered bumblebee}

From the revolving wing studied in the previous section we now proceed to the case of a bumblebee. A key advantage of our numerical method is the simplicity with which complex geometries can be taken into account. Therefore we include the insect's body in the computational model, including its legs, antennae and proboscis. For an illustration we refer to Fig. 1 (c). The body is responsible for the major part of aerodynamic drag and it may contribute, though less significantly, to the lift as well. In the interest of brevity we refer to the supplementary material of [9] for a complete description of the modeled insects morphology.

Fig. 8 illustrates, in the top and bottom strips, the wingbeat kinematics for the down- and upstroke. The mean stroke plane is inclined with respect to horizontal, and the geometric angle of attack is larger during the downstroke.

Fig. 8 also shows visualizations of the flow field at two selected instants, $t=0.3$ and $t=0.7$, which are in the middle of the down- and upstroke, respectively. The vorticity field, $|\boldsymbol{\omega}|$, shows the large amount of vorticity generated at the wing's leading edges (A). This zone of intense vorticity appears to be continuous even in the tip vortex. Behind the body, where the wings shed their leading edge vortices at the end of the previous upstroke (B), another zone with elevated values of vorticity exists. The overall flow topology is highly complex, but symmetry is not broken. The reason for this symmetry lies in the precision of the numerical method, in which no symmetrybreaking perturbations occur. The visualization of helicity however shows that leading edge and tip vortex can be clearly distinguished (C) as they have opposite signs in $H$. This distribution of vorticity and helicity is qualitatively similar to what has been found in the case of the revolving wing in Fig. 2, though in the quantitative scales of $H$ and $|\boldsymbol{\omega}|$ differ significantly, even for a comparable Reynolds number. However the Reynolds number is difficult to compare in both cases, as the wingtip velocity in the bumblebee case is not constant. Instead, the cycle-averaged value is used, but this implies that the instantaneous wingtip velocity can be larger than in the revolving case. In addition, the mean flow, which was not present in the revolving wing, increases the instantaneous relative velocity during the downstroke, as the wings move upstream.

Remarkably, many regions containing vorticity away from the insects exhibit less helicity, cf. $\mathrm{D}_{1}$ and $\mathrm{D}_{2}$. As discussed previously, this implies that the non-linearity of the Navier-Stokes equation is strong in those regions, and that these structure participate more in the Kolmogorov cascade of energy.

At the end of the downstroke, the wing reverses its direction, and the leading edge vortex is shed into the wake. The resulting vortex 'puff' can be seen in the second visualized time instant (E). The puff features a much more complex topology than the leading edge vortex (A), and its helicity has no preferential sign. A new, though weaker leading edge vortex is formed (F) at the wings, with the same pattern of helicity and vorticity. The wingtip vortex from the downstroke has formed two vortex filaments that form a helix $(G)$. 


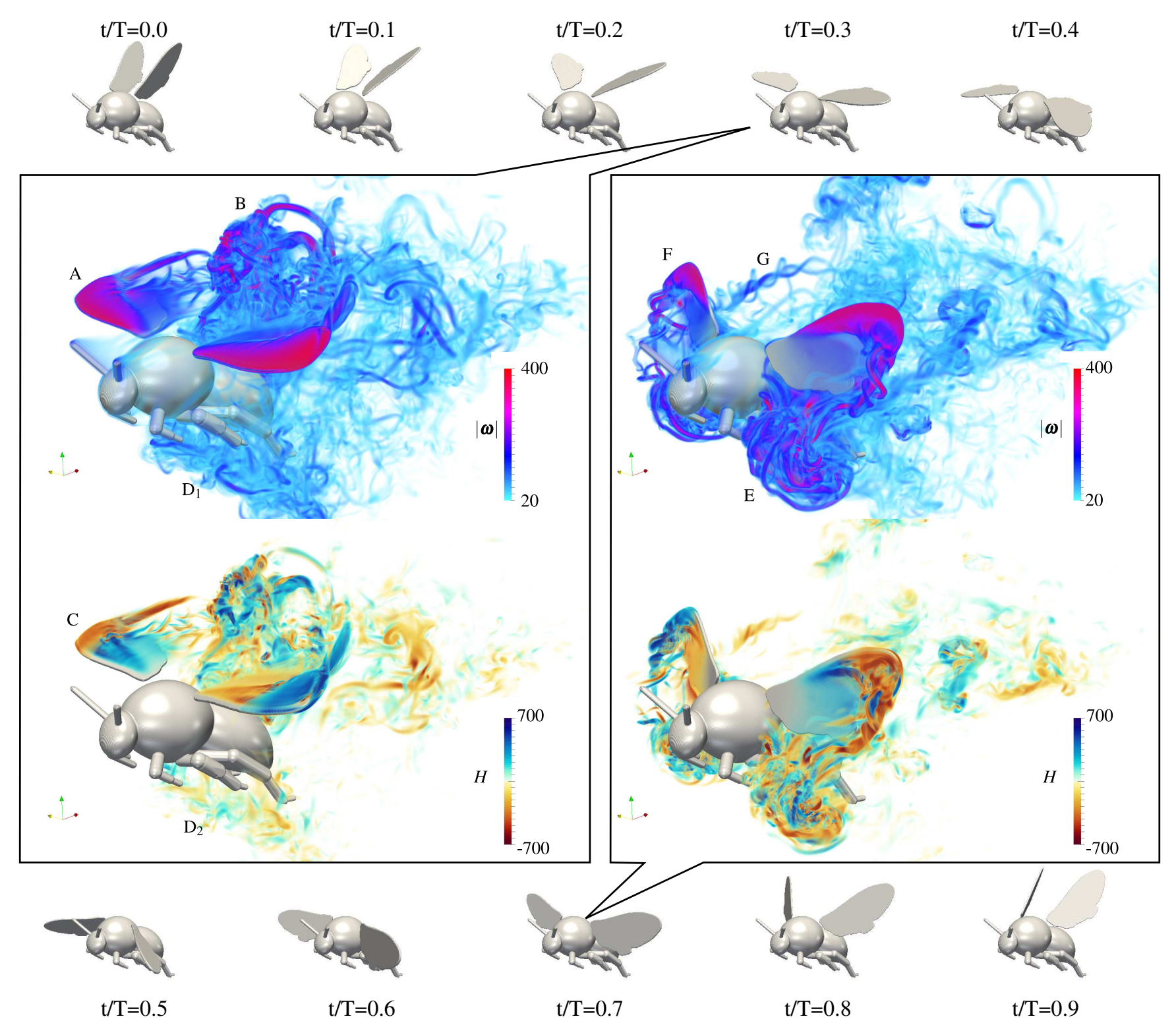

Figure 8: Bumblebee in laminar inflow. Shown is the wingbeat kinematics for the down- (top) and upstroke (bottom). For two selected times during down- and upstroke, the flowfield is visualized by vorticity magnitude (top) and helicity (bottom). Helical leading edge vortices can be identified.

We thus note that the LEV has similar features to the one produced in a rotating wing, but the wake topology differs due to the reciprocal flapping motion.

We now proceed and revisit the model bumblebee in turbulent inflow, in the same manner as has been done in previous work [9]. The inflow turbulence, imposed in a layer upstream of the insect, consists of velocity fluctuations $\boldsymbol{u}^{\prime}$ added to the mean inflow $\boldsymbol{u}_{\infty}$. The fluctuations are obtained from pre-computed simulations of homogeneous isotropic turbulence (HIT) with Reynolds numbers $R_{\lambda}$ ranging 90 to 230 . Scaled to the insect dimensions this yields turbulence intensities $T u=u_{\mathrm{RMS}}^{\prime} / u_{\infty}$ between 0.17 and 0.99 . For all turbulence intensities, a single realization is, due to the erratic nature of turbulence, not fully representative. Thus, several realizations for each 
turbulence intensity have been computed. The number of independent wingbeats available for averaging varies between 16 for the lowest and 108 for the largest value of $T u$.

The main result of [9] was that the ensemble-averaged forces, torques and the aerodynamic power did not differ from the values in the laminar case, though the values fluctuated of course. It was concluded that even in the strongest background turbulence, no systematic destruction of the leading edge vortex occured, as this would have resulted in a significant change in the aerodynamic quantities.

In the present work, the emphasis lies on the helicity, which we did not consider previously. Integrating the helicity over the half-space of the computational domain with respect to the bilateral symmetry plane of the insect, one obtains the mean helicity generated by the left and the right wing. The top part of Fig. 9 shows the left- and right wing contribution for two individual realizations. The black line corresponds to laminar inflow, and the integral helicity is symmetric except for the sign. Their sum is therefore zero, meaning that the bumblebee produces no net helicity in the wake. By contrast, the orange line corresponds to a single realization of $T u=0.99$. The strong inflow turbulence breaks the symmetry, and thus, even though the HIT fields do not contain a net helicity, the left- and right wing contributions do not add to zero.

The bottom part of Fig. 9 shows time evolutions of the ensemble-averaged values of left- and right helicity. The black line again corresponds to the laminar inflow. The values in the turbulent simulations however are similar to the laminar ones. This finding is consistent with [9]. The standard-deviation of the helicity grows with $T u$ increasing, thus higher $T u$ implies, as expected, larger fluctuations.

\section{Conclusion}

By means of high resolution direct numerical simulations we studied two flow configurations relevant to insect flight. First, a rotating bumblebee wing at two Reynolds numbers has been considered as canonical problem, then we passed to a compete bumblebee model, in order to check if the results obtained in the former can be extrapolated to the latter.

The revolving wing has been considered at two Reynolds numbers, based on the wingtip velocity, of about 2000 and 200. A leading edge and tip vortex is observed in both cases. We found that helicity is not produced near the leading edge, but instead at a position towards the trailing edge, and that it is due to the axial flow generated by the pressure deficit at the wing tip. This flow does not develop immediately at the leading edge, hence the lack of helicity there. The vortex core is highly helical, with large values of $H$, and $h$ near unity, corresponding thus to alignment or anti-alignment of velocity and vorticity. The nonlinear term is therefore depleted, and the leading edge vortex can be interpreted as a coherent structure as proposed in the literature. This finding is

important as it provides a complementary point of view on the observed stability of the LEV, not in contradiction to other concepts like the axial transport of excess vorticity [34]. An analysis with 

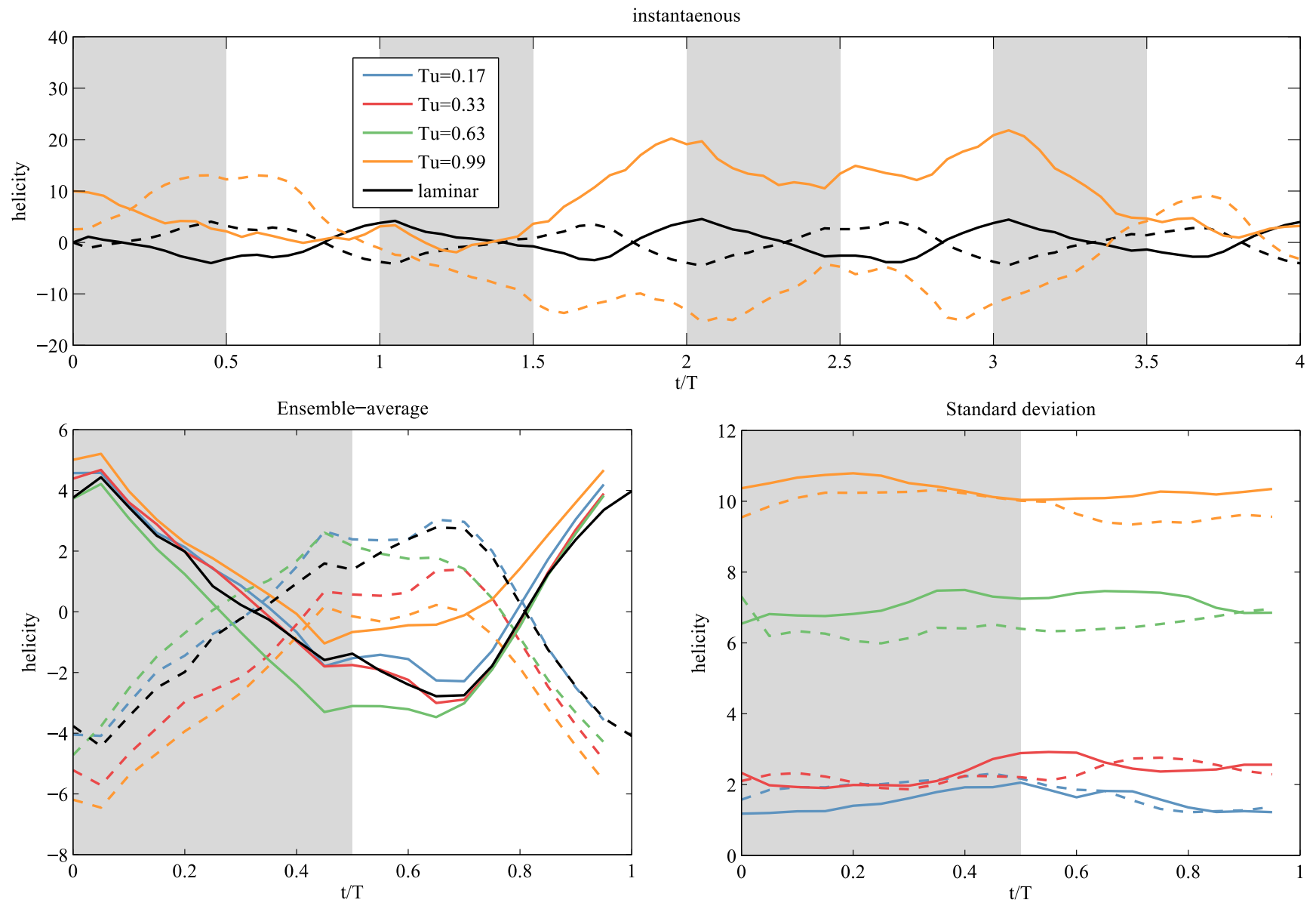

Figure 9: Bumblebee for laminar inflow and turbulent inflow with turbulence intensity $T u=$ $0.17,0.33,0.63$ and 0.99 . Top: time evolution of the instantaneous helicity $H$ integrated over the left and right domain with respect to the vertical center plane of the bumblebee. Bottom: time evolution of the ensemble averaged helicity $H$ integrated over the left and right domain with respect to the vertical center plane of the bumblebee for laminar inflow and turbulent inflow (left) and corresponding standard deviation (right).

orthogonal wavelets allowed us to characterize the most helical scale and its spatial intermittency. We showed, in agreement with experimental results, that the integral helicity on the top side of the wing is sensitive to the Reynolds number, and exhibits, at the higher Re considered, a significant drop that can be interpreted as vortex bursting. The aerodynamic force production was indeed not affected by this bursting, and the burst LEV remained attached to the wing.

We verified then, using the bumblebee model, that these results can be extrapolated to real insects with their more complex flapping motion, as opposed to the simple, continuous rotation. Similar features in the flow were found, namely helical LEVs and tip vortices with opposite helicity. In addition, turbulent inflow has been imposed, and we confirmed, in agreement with [9], that turbulence does not alter ensemble-averaged flight characteristics, also regarding the helicity.

Acknowledgements. Financial support from the ANR (Grant 15-CE40-0019) and DFG (Grant SE 
$824 \backslash 26$-1), project AIFIT, is gratefully acknowledged and CPU time from the supercomputer center Idris in Orsay, project i20152a1664. For this work we were also granted access to the HPC resources of Aix-Marseille Université financed by the project Equip@Meso (ANR-10-EQPX-29-01). TE, KS, MF, FL and JS thankfully acknowledge financial support granted by the ministères des Affaires étrangères et du développement International (MAEDI) et de l'Education nationale de l'Enseignement supérieur et de la Recherche (MENESR), and the Deutscher Akademischer Austauschdienst (DAAD) within the FrenchGerman Procope project FIFIT. DK gratefully acknowledges the financial support from the JSPS (Japan Society for the Promotion of Science) Postdoctoral Fellowship, JSPS KAKENHI Grant Number 15F15061.

\section{References}

[1] P.S. Addison. The Illustrated Wavelet Transform Handbook: Introductory Theory and Applications in Science, Engineering, Medicine and Finance. Taylor \& Francis, London, 2002.

[2] P. Angot, C. Bruneau, and P. Fabrie. A penalization method to take into account obstacles in incompressible viscous flows. Numer. Math., 81:497-520, 1999.

[3] R. Betchov. Semi-isotropic turbulence and helicoidal flows. Phys. Fluids, 4:925-926, 1961.

[4] D. Chen, D. Kolomenskiy, H. Liu. Closed-form solution for the edge vortex of a revolving plate. J. Fluid Mech. 821:200-218, 2017.

[5] J. D. Crall, J. J. Chang, R. L. Oppenheimer and S. A. Combes. Foraging in an unsteady world: bumblebee flight performance in field-realistic turbulence. Interface Focus, 7(1), 2016.

[6] C. P. Ellington, C. van den Berg, A. P. Willmott and A. L. R. Thomas. Leading edge vortices in insect flight. Nature, 384, 626, 1996.

[7] C. P. Ellington. The Aerodynamics of Hovering Insect Flight. 1. The Quasi-Steady Analysis. Phil. Trans. R. Soc. Lond. B, 305:1-15, 1984.

[8] C.P. Ellington. The novel aerodynamics of insect flight: applications to micro-air vehicles. J. Exp. Biol., 202:3439-3448, 1999.

[9] T. Engels, D. Kolomenskiy, K. Schneider, F.O. Lehmann and J. Sesterhenn. Bumblebee flight in heavy turbulence. Phys. Rev. Lett., 116, 028103, 2016.

[10] T. Engels, D. Kolomenskiy, K. Schneider and J. Sesterhenn. FluSI: A novel parallel simulation tool for flapping insect flight using a Fourier method with volume penalization. arXiv:1506.06513, 2015. SIAM J. Sci. Comput., 38(5), S3-S24, 2016.

[11] M. Farge. Wavelet transforms and their applications to turbulence. Annu. Rev. of Fluid Mech., 24, 395-457 (1992). 
[12] M. Farge, G. Pellegrino and K. Schneider. Coherent vortex extraction in 3D turbulent flows using orthogonal wavelets. Phys. Rev. Lett., 87 (5), 054501, 2001.

[13] M. Farge and K. Schneider. Wavelet transforms and their applications to MHD and plasma turbulence: a review. J. Plasma Phys., 81(6), 435810602, 2015, arXiv:1508:05650.

[14] D. J. Garmann and M. R. Visbal. Dynamics of revolving wings for various aspect ratios. J. Fluid Mech., 748:932-956, 2014.

[15] R. R. Harbig, J. Sheridan, and M. C. Thompson. Reynolds number and aspect ratio effects on the leading-edge vortex for rotating insect wing planforms. J. Fluid Mech., 717:166-192, 2013.

[16] K. Minami, K. Suzuki and T. Inamuro. Free flight simulations of a dragonfly-like flapping wing-body model using the immersed boundary-lattice Boltzmann method. Fluid Dyn. Res, 47:015505, 2015.

[17] A. R. Jones, A. Medina, H. Spooner, and K. Mulleners. Characterizing a burst leading-edge vortex on a rotating flat plate wing. Exp. Fluids, 57:52, 2016.

[18] D. Kolomenskiy and K. Schneider. A Fourier spectral method for the Navier-Stokes equations with volume penalization for moving solid obstacles. J. Comput. Phys., 228:5687-5709, 2009.

[19] D. Kolomenskiy, M. Maeda, T. Engels, H. Liu, K. Schneider and J.-C. Nave. Aerodynamic ground effect in fruitfly sized insect takeoff. arXiv:1504.04484. PLoS ONE, 11(3): e0152072, 2016.

[20] D. Kolomenskiy, H.K. Moffatt, M. Farge and K. Schneider. Two- and three-dimensional numerical simulations of the clap-fling-sweep of hovering insects. J. Fluids Struct., 27, 784-791, 2011.

[21] D. Kolomenskiy, H.K. Moffatt, M. Farge and K. Schneider. The Lighthill-Weis-Fogh clapfling-sweep mechanism revisited. J. Fluid Mech., 676, 572-606, 2011.

[22] D. Kolomenskiy, T. Engels and K. Schneider. Numerical Modelling of Flexible Heaving Foils. J. Aero Aqua Bio-mechanisms, 3, 22-28, 2013.

[23] D. Kolomenskiy, Y. Elimelech and K. Schneider. Leading-edge vortex shedding from rotating wings. Fluid Dyn. Res., 46, 031421, 2014.

[24] S. Kurien, M. A. Taylor, and T. Matsumoto. Cascade time scales for energy and helicity in homogeneous isotropic turbulence. Phys. Rev. E, 69,6, 066313, 2004 
[25] Y. J. Lee, K. B. Lua and T. T. Lim. Aspect ratio effects on revolving wings with Rossby number consideration. Bioinspir. Biomim., 11(5), 2016.

[26] F.O. Lehmann and S. Pick. The Aerodynamic benefit of wing--wing interaction depends on stroke trajectory in flapping insect wings. J. Exp. Biol., 210:1362--1377, 2008

[27] D. Lentink, W.B. Dickson, J.L. Van Leeuwen and M.H. Dickinson. Leading-edge vortices elevate lift of autorotating plant seeds. Science, 324(5933), pp.1438-1440, 2009.

[28] D. Lentink and M.H. Dickinson. Rotational accelerations stabilize leading edge vortices on revolving fly wings. J. Exp. Biol., 212:2705--2719, 2009.

[29] P. B. S. Lissaman. Low-Reynolds-number airfoils. Annu. Rev. Fluid Mech., 15(1):223-239, 1983.

[30] H. Liu and K. Kawachi. A numerical study of insect flight. J. Comput. Phys., 146:124-156, 1998

[31] H. Liu, C.P. Ellington, K. Kawachi, C. Van Den Berg and A.P. Willmott. A computational fluid dynamic study of hawkmoth hovering. J. Exp. Biol., 201(4), 461-477, 1998.

[32] K. B. Lua, T. T. Lim, and K. S. Yeo. Scaling of aerodynamic forces of three-dimensional flapping wings. AIAA Journal, 52(5):1095-101, 2014.

[33] T. Maxworthy Experiments on the Weis-Fogh mechanism of lift generation by insects in hovering flight: I. Dynamics of the []fling. J. Fluid Mech., 93, 47-63, 1979.

[34] T. Maxworthy The formation and maintenance of a leading-edge vortex during the forward motion of an animal wing. J. Fluid Mech., 587, 471-475, 2007.

[35] C. Meneveau. Analysis of turbulence in the orthonormal wavelet representation. J. Fluid Mech., 232, 469, 1991.

[36] H.K. Moffatt. The degree of knottedness of tangled vortex lines. J. Fluid Mech. , 35, 117-129, 1969.

[37] H.K. Moffatt and A. Tsinober. Helicity in laminar and turbulent flow. Annu. Rev. Fluid Mech., 24, 281-312, 1992.

[38] H.K. Moffatt. Helicity and singular structures in fluid dynamics. Proc. Natl. Acad. Sci. U. S. A., 111(10), 3663-3670, 2014

[39] J. J. Moreau. Constantes d'un îlot tourbillonnaire en fluid parfait barotrope. C.R. Acad. Sci. Paris, 252, 2810-2812, 1961 
[40] S. Pick and F.-O. Lehmann. Stereoscopic PIV on multiple color-coded light sheets and its application to axial flow in flapping robotic insect wings. Exp. Fluids, 47(6), 1009, 2009.

[41] S. Ravi, J.D. Crall, A. Fisher, and S. A. Combes. Rolling with the flow: bumblebees flying in unsteady wakes. J. Exp. Biol., 216:4299-4309, 2013.

[42] K. Schneider. Numerical simulation of the transient flow behaviour in chemical reactors using a penalisation method. Computers $\&$ Fluids, 34:1223-1238, 2005.

[43] T. Weis-Fogh. Quick estimates of flight fitness in hovering animals, including novel mechanisms for lift production. J. Exp. Biol., 59:169--230, 1973.

[44] T. J. Wolf, C. P. Ellington, and I. S. Begley. Foraging costs in bumblebees: field conditions cause large individual differences. Insectes Sociaux, 46(3):291-295, 1999.

[45] J. R. Usherwood and F.-O. Lehmann. Phasing of dragonfly wings can improve aerodynamic efficiency by removing swirl. J. R. Soc. Interface, 5, 1303-1307, 2008.

[46] K. Yoshimatsu, N. Okamoto, K. Schneider, Y. Kaneda and M. Farge. Intermittency and scale-dependent statistics in fully developed turbulence. Phys. Rev. E, 79, 026303, 2009. 